\title{
Assessing the impacts of cell wall composition on the optimum stage for "Uradome" in moso bamboo
}

Yuka Furusawa ${ }^{1,2^{*}}$ and Tatsuya Ashitani $i^{1,3}$

\begin{abstract}
Snow damage is problematic when cultivating bamboo shoots, and "Uradome", the practice of removing the tips of new shoots, is used as a preventative measure. Producers perform "Uradome" at empirical times, but there is no scientific basis for this. We hypothesized that differences in the structure and composition of the cell wall might affect the optimal timing of "Uradome" and analyzed the cell wall components of the "Uradome" portion. The lower plant sections broken by the "Uradome" had larger cellulose and lignin depositions than the upper sections. However, there were no differences in the lignin structure or ratio between the upper and lower nodes of the broken sections. This suggests that differences in the degree of cellulose and lignin deposition have significant effects on "Uradome" sites, and that the timing coincides with the development of one or two juvenile branches, which growers empirically consider to be the appropriate time. These results are considered to be new findings that scientifically support the cultivation management of bamboo that has been conducted empirically.
\end{abstract}

Keywords: Moso bamboo, "Uradome", Cell wall, Cellulose, Lignin

\section{Introduction}

There are approximately $80-90$ genera and $1000-1500$ species of bamboo in the world, and they are biogeographically classified into tropical and temperate groups [1]. In the temperate zone, they are distributed in China, Chile, the United States, and Japan [2], and the largest species of bamboo in Japan is moso bamboo (Phyllostachys pubescens) [3]. It was introduced to Japan from China approximately 300 years ago, and the young shoots or bamboo shoots, can be consumed as a food source [3].

Bamboo shoots are currently cultivated across Japan, including snow-covered areas [4]. One of the major problems of growing bamboo shoots in snow-covered areas, however, is the occurrence of snow damage, as it can negatively affect their growth in the following years.

\footnotetext{
*Correspondence: furusaway@pref.yamagata.jp

${ }^{1}$ The United Graduate School of Agricultural Sciences, Iwate University,

3-18 Ueda, Morioka, Iwate 020-0066, Japan

Full list of author information is available at the end of the article
}

Snow damage in bamboo shoots has been investigated previously in both Japan and China [5]. Investigations of the management methods used to prevent snow damage identified that "Uradome" was an effective measure [6]. "Uradome" is the act of removing the tip of a bamboo shoot by breaking or cutting it after it has grown to become bamboo. It is conducted either with a blade or by shaking the trunk back and forth to break the tip by recoil [7]. Since the use of a blade can be dangerous, many growers use the latter method of shaking off the tip.

Based on the experience of growers, the optimal time for "Uradome," when using the shake off method, is when there are one or two young branches [7, 8]. The date of application varies from stem to stem and there are only a few days during the bamboo growing season that it can be applied. If the practice is conducted earlier than the appropriate date, the trunk will be die by excessive breakage from the lower nodes. On the other hand, if it is conducted later, it will not be possible to break the planned nodes and the effectiveness of preventing snow damage 
will be reduced. However, there is no scientific evidence explaining the correct time for this practice.

The authors hypothesized that differences in the cell wall structure and composition of the upper and lower parts of the nodes broken by the shake off method would affect the determination of the optimal "Uradome" time. To verify this, the main components and lignin structures of the cell walls in the broken parts were analyzed. In addition, the cell walls of the early stage bamboo growth, prior to the "Uradome" period and the cell walls of later stage bamboo growth, after the "Uradome" period, were analyzed and compared in the same manner.

\section{Materials and methods}

\section{Collection and storage of materials}

The materials were collected in a moso bamboo forest in Yamanobe Town, Yamagata Prefecture. Bamboo that was the optimal age for "Uradome" was used for this study, early-growth bamboo and first-year bamboo that has stopped elongation were used for comparison. Bamboo that was at the optimal age for "Uradome" was new bamboo that occurred in 2018, and "Uradome" was implemented in mid-June when the young branches began to emerge. The number of samples was three, and they were randomly selected from the bamboo forest. After implementation, the bamboo was collected just above, just below, and four nodes below the broken part (Fig. 1a). One sample of bamboo shoots at the early stage of growth (height: $1.5 \mathrm{~m}$ ) was collected in late May by random sampling. After collection, the most basal part of the ground was designated as one node and the uppermost part as 15 nodes, and bamboo shoot was cut into 14 parts. The part above the 15th node where it was no longer distinct was treated as one part (Fig. 1b). One of the current year's bamboos was collected by random sampling in mid-December (Fig. 1c). All materials were frozen immediately after collection and freeze-dried samples were prepared before analysis.

\section{Analysis of the cell wall compositional components}

The samples used to analyze the major cell wall components were as follows: bamboo at the optimal age for "Uradome": nodes 28, 31, and 32 (sample1), nodes 30, 33 and 34 (sample2), nodes 30, 33 and 34 (sample3) (Fig. 1a); bamboo in the early stage of growth: node 10 (Fig. 1b); and current year bamboo: node 10 (Fig. 1c). a Uradome Stage

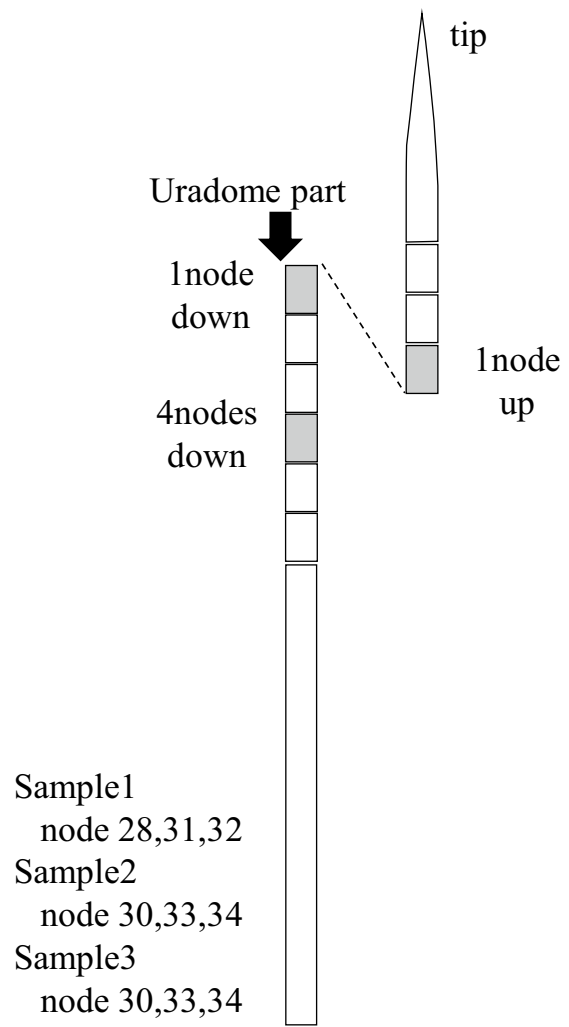

c Bamboo of current year

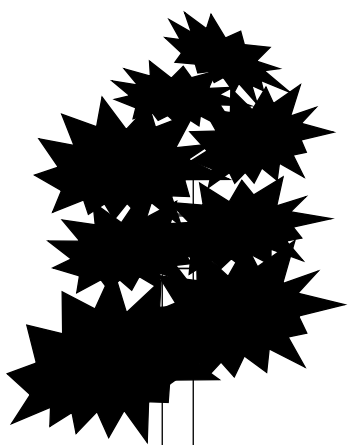

b early stages of growth

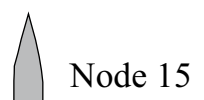

Node 10

Node 10

Node 2

Fig. 1 Nodes used in the experiments at each growth stage. The nodes in the gray area were used for the experiment 
Quantification of the cell wall components was performed according to standard method [9], and the test was repeated three times for each sample. The samples were extracted using an ethanol-benzene mixture (1:2) in a Soxhlet extractor according to the standard method. The obtained sample was used for the determination of holocellulose using the Wise method. The amount of hemicellulose was calculated by subtracting the amount of $\alpha$-cellulose from the amount of holocellulose. Lignin was determined using the Klason method, and acid-soluble lignin was determined by measuring the maximum absorbance around 205-210 nm with UV-vis spectrometer (UV1800, Shimadzu Corporation, Kyoto, Japan).

\section{Pyrolysis gas chromatography (Py-GC) analysis}

The samples used for the Py-GC analysis were as follows: bamboo at the optimal age for "Uradome": nodes 28,31 , and 32 (sample1), nodes 30,33 and 34 (sample2), nodes 30, 33 and 34 (sample3) (Fig. 1a); bamboo in the early stage of growth: nodes 2, 10, and 15 (Fig. 1b); and current year bamboo: node 10 (Fig. 1c). Approximately $0.6 \mathrm{mg}$ of each sample was wrapped in pyrofoil (Japan Analytical Industry F500), set in a Curie point pyrolysis injector (Japan Analytical Industry JIC-22), and subjected to Gas Chromatograph-Mass Spectrometer (GC-MS) (GCMS-QP2010Ultra) (Shimadzu Corporation, Kyoto, Japan) under the following pyrolysis conditions: $500{ }^{\circ} \mathrm{C}$ (5 s); GC-MS conditions: Rtx-5MS capillary column used, injection temperature: $250{ }^{\circ} \mathrm{C}$, column temperature: $50{ }^{\circ} \mathrm{C}(1 \mathrm{~min})$ to $320^{\circ} \mathrm{C}(5 \mathrm{~min})$, interface temperature: $300^{\circ} \mathrm{C}$, acquisition mass range: $50-800$ atomic mass units, carrier gas: helium. The peaks on the resulting chromatogram were identified by comparison with previous literature [10].

\section{Results and discussion}

\section{Compositional components of the cell wall}

The results of the analysis of the major components in the cell wall according to growth stage are shown in Table 1. For bamboo in the "Uradome" period, the mean value of three samples is shown. The bamboo at the optimal age for "Uradome" was approximately $30 \%$ alpha cellulose, $40 \%$ hemicellulose, $10 \%$ Klason lignin, and 5\% acidsoluble lignin. The alpha cellulose, hemicellulose, and Klason lignin values were higher than those for the earlygrowth bamboo, while the acid-soluble lignin was lower (Table 1). While, when compared with the results of the current year bamboo, the alpha cellulose and Klason lignin were lower, and the hemicellulose and acid-soluble lignin were higher in the "Uradome" bamboo (Table 1).

These results suggest that alpha cellulose increases with growth (early growth $<$ "Uradome" stage $<$ current year) and that the degree of cellulose deposition at the "Uradome" stage is intermediate, and between that of the early-growth and current-year bamboo. In addition, Klason lignin, which was not found in the early stage of growth, was found in the "Uradome" stage, although its composition was as low as $10 \%$, suggesting that the polymerization of lignin had progressed. However, the Klason lignin in the current year's bamboo was $30 \%$, suggesting that the "Uradome" stage was the initial stage of lignin deposition, and that lignin deposition would increase during the six months from mid-June to mid-December.

It has been reported that at 36-84 days after shoot growth, lignin deposition and polymerization occur simultaneously with an increase in the proportion of Klason lignin and a decrease in the proportion of acid-soluble lignin [11]. The "Uradome" stage was considered to correspond to the first half of the period reported. It has also been reported that in Sinobambusa tootsik, elongation growth started in May and ended in late June, and that the lignin content was approximately half of that of mature culms [12]. The lignin content results in this study were similar, although the species were different.

The compositional ratios of the main components of the upper and lower nodes broken during the shaking down method of the "Uradome" process are shown in Fig. 2. The results of the analysis of the three samples approximately showed a similar trend (Fig. 2a-c). Alpha

Table 1 Composition of major components of bamboo at each growth stage

\begin{tabular}{|c|c|c|c|c|c|}
\hline \multirow[t]{2}{*}{ Growth stage } & \multirow[t]{2}{*}{ Node } & \multicolumn{4}{|c|}{ Composition ratio $(\% \pm \mathrm{SE})$} \\
\hline & & a-cellulose & Hemicellulose & Klason lignin & ACID-soluble lignin \\
\hline \multirow[t]{3}{*}{ Uradome (average of 3 samples) } & 1 node up & $32.55 \pm 0.58$ & $35.92 \pm 0.36$ & $7.56 \pm 0.23$ & $4.32 \pm 0.18$ \\
\hline & 1 node down & $31.56 \pm 0.59$ & $35.97 \pm 0.85$ & $7.57 \pm 0.53$ & $4.08 \pm 0.18$ \\
\hline & 4 nodes down & $36.06 \pm 0.49$ & $38.83 \pm 0.43$ & $10.58 \pm 0.18$ & $2.95 \pm 0.10$ \\
\hline Early stages of growth & 10 & $24.14 \pm 0.72$ & $23.77 \pm 2.28$ & 0.00 & $13.81 \pm 0.14$ \\
\hline Bamboo of current year & 10 & $41.65 \pm 0.57$ & $29.89 \pm 0.72$ & $29.64 \pm 0.09$ & $1.46 \pm 0.04$ \\
\hline
\end{tabular}

Comparison of the compositional ratios of the major components at each growth stage. Bar: \pm SE 


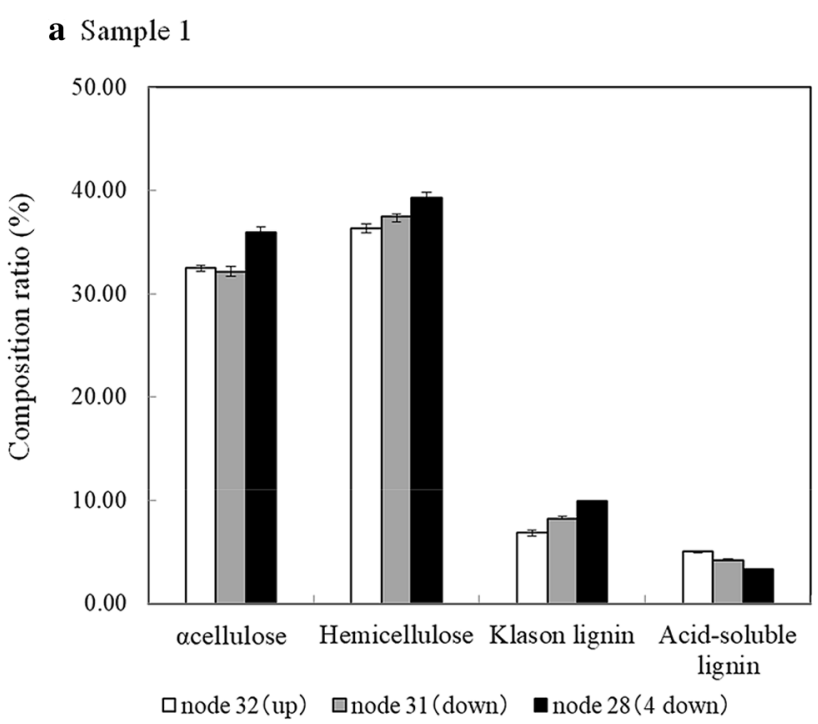

b Sample 2

c Sample 3
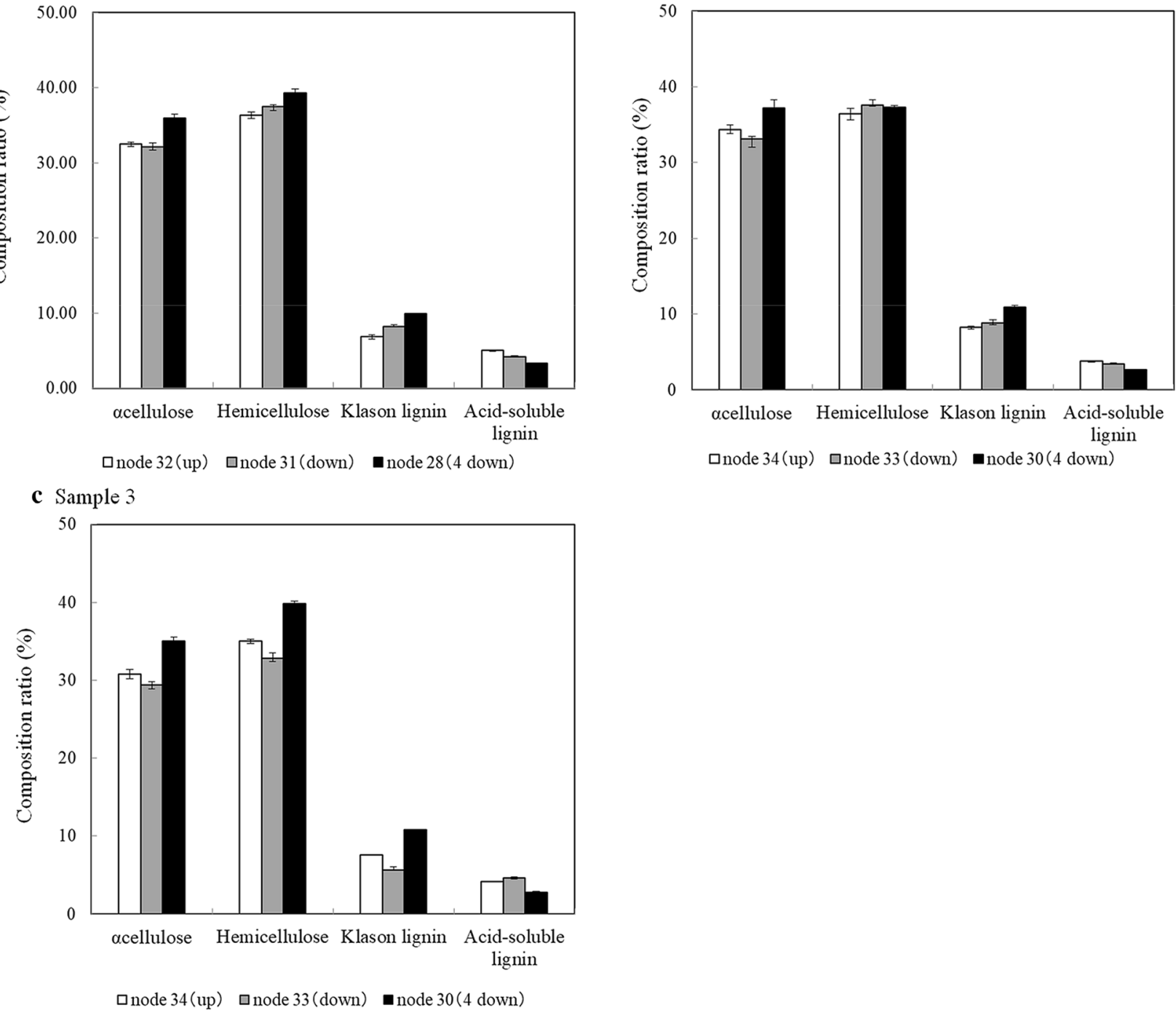

Fig. 2 Compositional ratios of the major components in the "Uradome" upper and lower sections. Compositional ratios of the major components of the upper and lower "Uradome" sections of the three samples. a sample1, b sample2, c sample3. Bar: \pm SE

cellulose, hemicellulose, and Klason lignin were more abundant in the order: up $<$ down $<4$ down, and the composition ratio tended to be larger in the lower nodes of the broken parts. On the other hand, acid-soluble lignin was more abundant in the order: 4 down $<$ down $<$ up, and the composition ratio tended to be larger in the upper nodes (Fig. 2a-c).

These results suggest that cellulose and lignin deposition in bamboo during the "Uradome" stage is more advanced in the lower nodes.

Cellulose crystallinity in the cell wall of moso bamboo was previously reported to gradually increase from the base to the top with the elongation of the internodes [13].
The present results were considered to be similar to this report. During the elongation of a $6 \mathrm{~m}$ bamboo, it was observed that L-tyrosine, a precursor of lignin, decreased rapidly around $3-4 \mathrm{~m}$, indicating that lignin is rapidly synthesized during growth [14]. The "Uradome" stage in this study was very close to that reported and it is possible that similar changes occurred above and below the sections affected by the "Uradome".

Cellulose crystallinity in the cell wall was found to increase outside the internodes of all the nodes of the bamboo culm, which is one of the countermeasures of bamboo culms against increased bending with elongation [13]. Lignin deposition and increase in the degree of 
polymerization may also be responsible for the increase in mechanical properties [11]. Since it has been reported that cellulose fibrils oriented in the axial direction of the cell wall maximize the longitudinal modulus of the fiber, and lignification increases the transverse stiffness [15], the differences in the degree of cellulose and lignin deposition in the nodes above and below the "Uradome" sections may have a significant effect on the mechanical actions during shake off.

\section{Analysis of lignin by Py-GC}

Py-GC analysis on the three samples from the "Uradome" period showed the same chromatograms for all three samples. As a result, pyrolysis products derived from GC-MS were detected (Fig. 3). The ratios of the $\mathrm{H}$, G, and S-types were approximately 55\%, 30\%, and $15 \%$, respectively (Fig. 4). The results were almost the same for the samples above and below the broken part.

The Py-GC results of the bamboo in the early-growth stage are shown in Fig. 5. As a result of the analysis, pyrolysis products derived from the $\mathrm{H}-$, G-, and S-types were detected in the second node near the ground. On the other hand, in sections 10 and 15, which were closer to the tip, only the $\mathrm{H}$ - and G-type pyrolysis products were detected. In section 10 , pyrolysis products derived from the G-type, which were not detected in section 15, were detected (Fig. 5). The ratio of H-, G-, and S-types was approximately $70 \%, 20 \%$, and $2 \%$, respectively, in section 2, indicating that there were more $\mathrm{H}$-types and fewer G- and S-types than that in the samples from the "Uradome" stage. The compositional ratios in sections 10 and 15 were almost the same, as they were more than $95 \%$ H-type and only a few G-types were identified (Fig. 6).

In addition, the Py-GC results for the current year's bamboo showed that pyrolysis products derived from the H-, G-, and S-types were detected (Fig. 7), and the composition ratio was approximately $40 \%, 50 \%$, and $10 \%$, respectively, with less $\mathrm{H}$-type and more G-type than in the samples from the "Uradome" stage, and the composition ratio of the S-type was about the same as that from the "Uradome" stage samples (Fig. 8).

In summary, the presence/absence and composition ratios of the pyrolysis products derived from the $\mathrm{H}-$, G-, and $\mathrm{S}$-types changed as growth progressed, with $\mathrm{H}$-types decreasing (early growth $>$ "Uradome" stage $>$ current year), G-types increasing (early growth $<$ "Uradome"

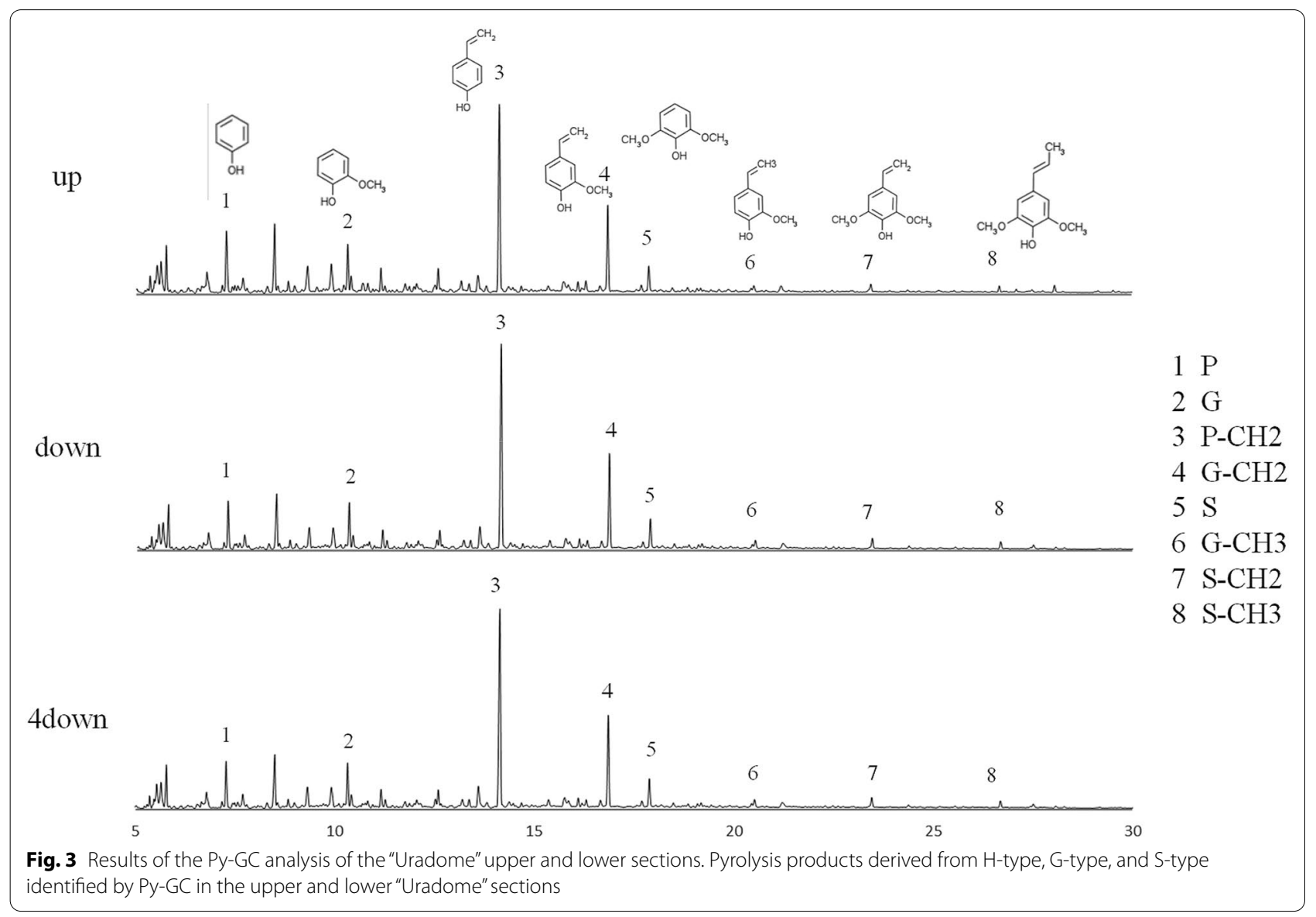



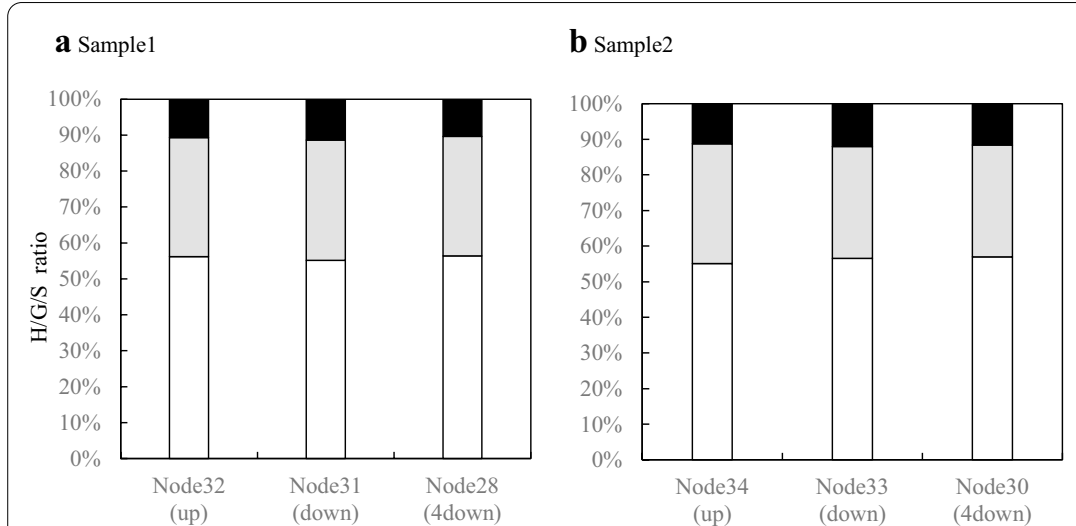

c Sample3

Fig. $4 \mathrm{H} / \mathrm{G} / \mathrm{S}$ ratio of lignin in "Uradome" period. H/G/S ratios of pyrolysis products of lignin in the upper and lower parts of the "Uradome" of three samples. a sample 1: nodes $28,31,32$, b sample 2: nodes 30, 33, 34, and c sample 3: nodes 30, 33,34

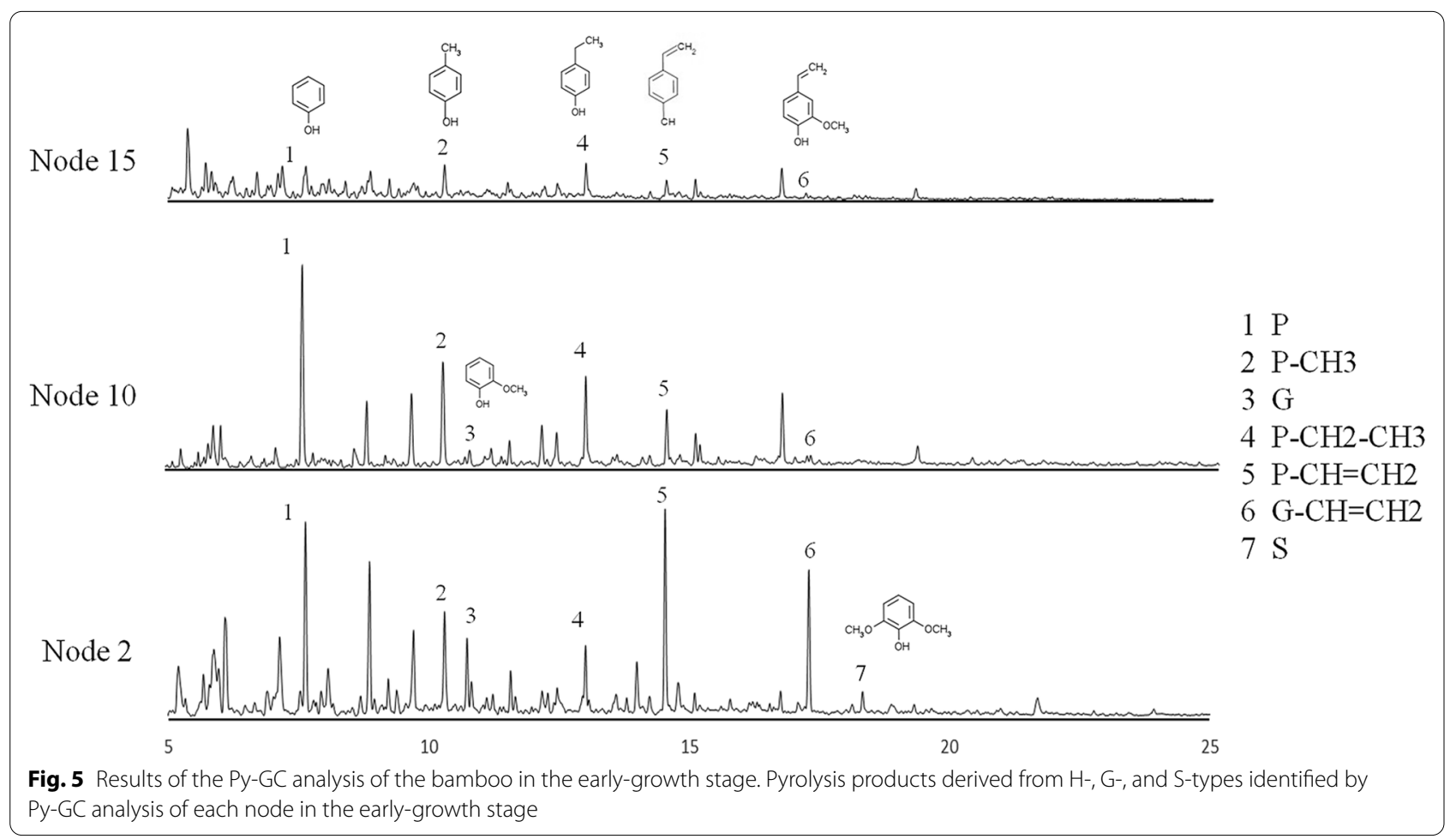

stage $<$ current year), and S-types increasing and then remaining constant (early growth<"Uradome" stage $=$ current year). In grasses, in addition to G- and S-type precursors, H-type precursors also contribute to polymerization [16], and the ratio of S- to G-type precursors has been reported to change during culm development [12], and the same trend was observed in this investigation. Previous reports have shown that S-, G-, and H-type lignin monomers were present in all samples from year 1 to year 6 [17]. In this study, we used samples from an earlier stage than reported there, enabling us to obtain results before the formation of S-type lignin monomers. Consequently, we were able to clarify the details for the increase and decrease processes of the three types of lignin monomers. It is said that lignin in the early stage of lignification is syringyl< guaiacyl, and the S-type increases as lignification progresses [2]. In the present study, however, S-type lignin increased only up to the "Uradome" stage and then remained constant, while G-type lignin tended to increase. The reason for the 


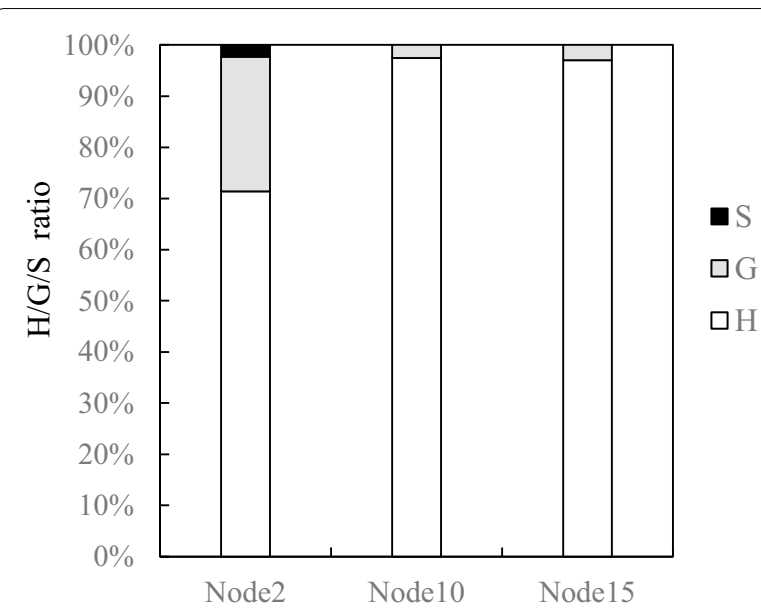

Fig. $6 \mathrm{H} / \mathrm{G} / \mathrm{S}$ ratio of lignin in the early-growth stage. H/G/S ratios of pyrolysis products of lignin in the early-growth stage. The samples were nodes 2,10 , and 15

different results cannot be clarified solely from the results of this study, and it requires further investigation. There were no differences in the pyrolysis products detected in the nodes above and below the broken part of the "Uradome", and the ratios of the pyrolysis products were similar. Therefore, the influence of the lignin structure on the determination of "Uradome" parts determined to be small.

\section{Conclusion}

The results of this study showed that the deposition of cellulose and lignin in the cell walls of the bamboo during "Uradome" was intermediate between that of the early stage of growth and that of the current year bamboo. In addition, cellulose and lignin deposition in the lower nodes of the broken sections, was more advanced than in the upper nodes. However, there was no difference in the lignin structures and ratios between the upper and

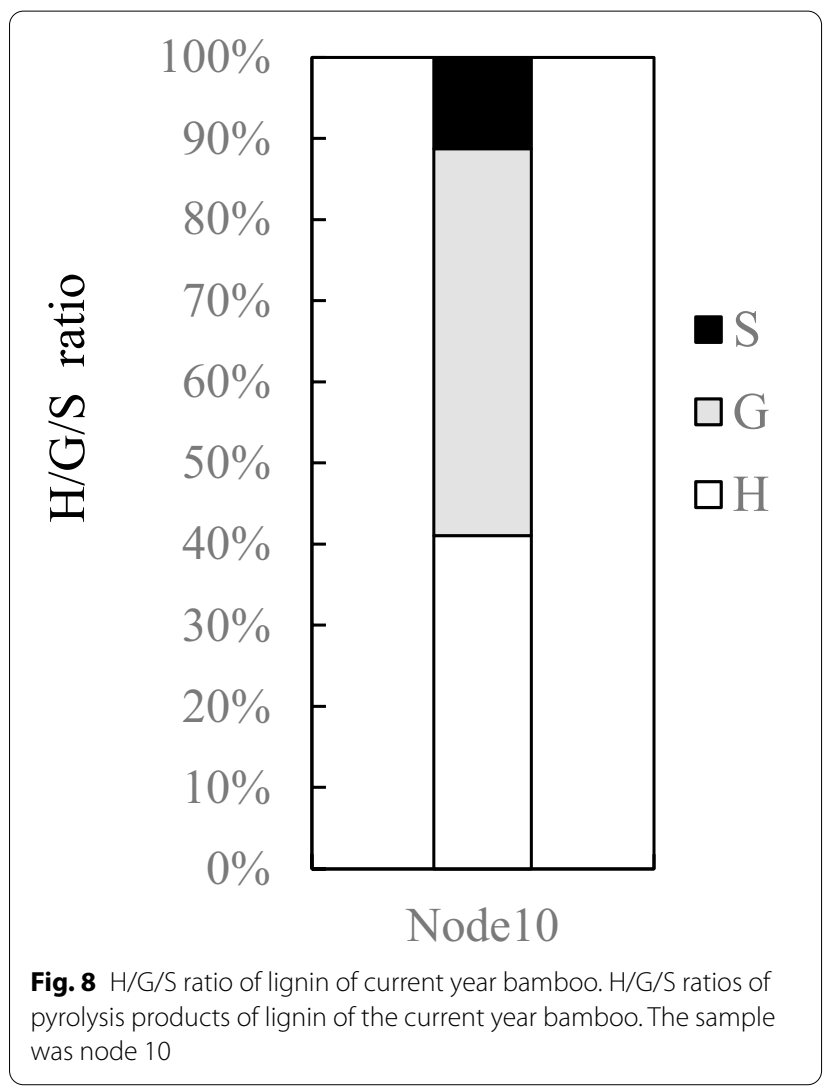

lower nodes of the "Uradome" broken sections. This suggests that differences in the degree of deposition for the cellulose and lignin have a significant effect on the determination of the "Uradome" sections. According to the empirical results of growers, the optimum time for "Uradome" is when one or two juvenile bamboo branches develop, as it coincides with changes in the cellulose and lignin depositions. These results are considered to be new findings that scientifically support the cultivation management of bamboo that has been conducted empirically.

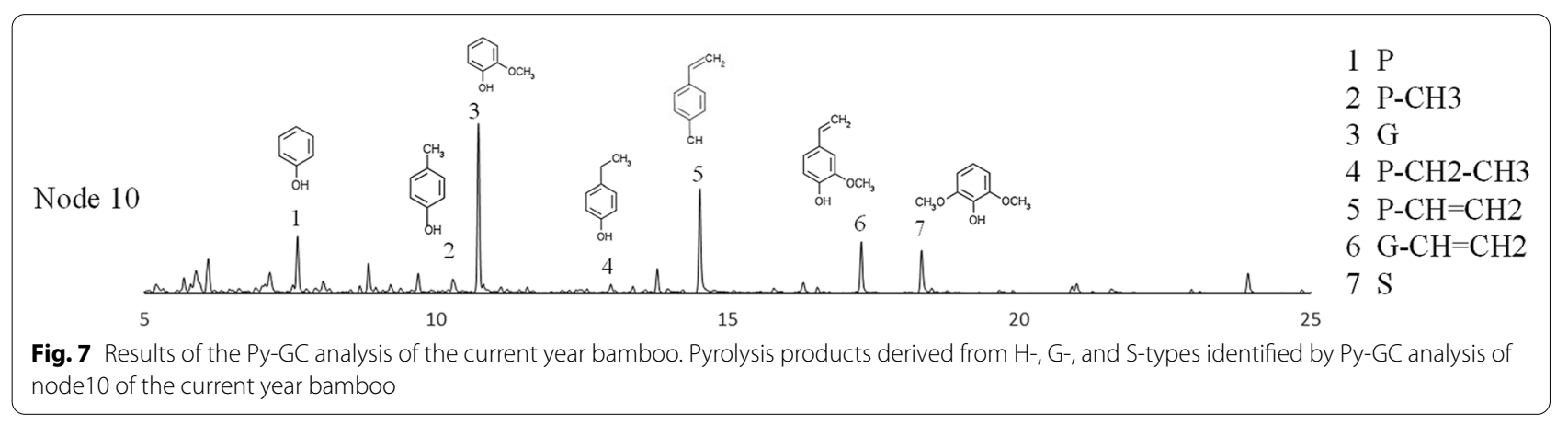




\section{Abbreviations}

Py-GC: Pyrolysis gas chromatography; GC-MS: Gas chromatograph-mass spectrometer; H-type: $p$-Hydroxyphenyl; G-type: Guaiacyl; S-type: Syringyl.

\section{Acknowledgements}

We would like to express our sincere gratitude to Mr. Akifumi Takauchi for his cooperation in the "Uradome" work and for providing samples for this study. We would also like to express our gratitude to Mr. Tomohiro Kubo for his cooperation in preparing the samples for analysis. The authors would like to thank Enago for the English language review.

\section{Authors' contributions}

YF was responsible for all experiments and writing; TA provided advice on chemical analysis and discussion. Both authors read and approved the final manuscript.

\section{Funding}

Not applicable.

\section{Availability of data and materials}

All data generated or analyzed during this study are included in this published article.

\section{Declarations}

\section{Competing interests}

The authors declare that they have no competing interests.

\section{Author details}

${ }^{1}$ The United Graduate School of Agricultural Sciences, Iwate University, 3-18 Ueda, Morioka, Iwate 020-0066, Japan. ${ }^{2}$ Yamagata Prefectural Forest Research and Instruction Center, 2707 Hei Sagae, Sagae, Yamagata 991-0041, Japan. ${ }^{3}$ Faculty of Agriculture, Yamagata University, 1-23 Wakabamachi, Tsuruoka, Yamagata 997-0037, Japan.

Received: 17 March 2021 Accepted: 7 May 2021

Published online: 13 May 2021

\section{References}

1. Sungkaew S, Stapleton CM, Salamin N, Hodkinson TR (2009) Non-monophyly of the woody bamboos (Bambuseae; Poaceae): a multi-gene region phylogenetic analysis of Bambusoideae ss. J Plant Res 122(1):95

2. Lin J, He X, Hu Y, Kuang T, Ceulemans R (2002) Lignification and lignin heterogeneity for various age classes of bamboo (Phyllostachys pubescens) stems. Physiol Plant 114(2):296-302
3. Shibata S (2003) Phyllostachys pubescens and Japanese (in Japanese). JSRT 28(3):406-411

4. Statistical survey on production of special forest products. https://www. maff.go.jp/j/tokei/kouhyou/tokuyo_rinsan/. Accessed 1 Feb 2021.

5. Zhou B, Li Z, Wang X, Cao Y, An Y, Deng Z, Letu G, Wang G, Gu L (2011) Impact of the 2008 ice storm on moso bamboo plantations in southeast China. J. Geophys Res 116:G00H06

6. Furusawa Y, Takahashi K, Ashitani T (2019) "Uradome" treatment for prevention of snow damage and terrain parameters of moso bamboo (Phyllostachys pubescens) forest in Tsuruoka, Yamagata Prefecture. J Sustain For 38(2):171-182

7. Ueda K (1963) Useful bamboo and bamboo shoot: new technology of cultivation. Hakubunkan Shinsha Publishers, Tokyo ((in Japanese))

8. Yamagata Prefecture (2017) Moso bamboo cultivation management manual (Yamagata Prefecture version). Yamagata Forest Research and Training Center, Yamagata

9. Nihon Mokuzai Gakkai (2000) Wood science laboratory manual. Buneido Publishers, Tokyo ((in Japanese))

10. Ralph J, Hatfield RD (1991) Pyrolysis-GC-MS characterization of forage materials. J Agric Food Chem 39(8):1426-1437

11. Kiryu T, Matsuda N, Kojiro K, Furuta Y (2017) The mechanism of improvement of physical properties of moso bamboo (Phyllostachys pubescens) with increasing age II. Mokuzai Gakkaishi 63(1):14-20 ((in Japanese))

12. Tsuyama T, Shimada N, Motoda T, Matsushita Y, Kijidani Y, Fukushima K, Kamei I (2017) Lignification in developing culms of bamboo Sinobambusa tootsik. J Wood Sci 63(6):551-559

13. Toba K, Nakai T, Shirai T, Yamamoto H (2015) Changes in the cellulose crystallinity of moso bamboo cell walls during the growth process by X-ray diffraction techniques. J Wood Sci 61(5):517-524

14. Fujii Y, Azuma Jl, Marchessault RH, Morin FG, Aibara S, Okamura K (1993) Chemical composition change of bamboo accompanying its growth. Holzforschung 47(2):109-115

15. Wang X, Ren H, Zhang B, Fei B, Burgert I (2012) Cell wall structure and formation of maturing fibres of moso bamboo (Phyllostachys pubescens) increase buckling resistance. J R Soc Interface 9:988-996

16. Tobimatsu Y (2017) Plant Cell wall: a crucial supporter of plant and human life. Seizonken kenkyu 13:10-18 ((in Japanese))

17. Zhu Y, Huang J, Wang K, Wang B, Sun S, Lin X, Song L, Wu A, Li H (2020) Characterization of lignin structures in Phyllostachys edulis (Moso bamboo) at different ages. Polymers 12(1):187

\section{Publisher's Note}

Springer Nature remains neutral with regard to jurisdictional claims in published maps and institutional affiliations.

\section{Submit your manuscript to a SpringerOpen ${ }^{\mathcal{O}}$ journal and benefit from:}

- Convenient online submission

- Rigorous peer review

- Open access: articles freely available online

- High visibility within the field

- Retaining the copyright to your article

Submit your next manuscript at $\boldsymbol{\nabla}$ springeropen.com 